\title{
The Relations among Measurements of Informant Discrepancies within a Multisite Trial of Treatments for Childhood Social Phobia
}

\author{
Andres De Los Reyes • Candice A. Alfano • \\ Deborah C. Beidel
}

Published online: 15 December 2009

(C) Springer Science+Business Media, LLC 2009

\begin{abstract}
Discrepancies between informants' reports of children's behavior are robustly observed in clinical child research and have important implications for interpreting the outcomes of controlled treatment trials. However, little is known about the basic psychometric properties of these discrepancies. This study examined the relation between parent-child reporting discrepancies on measures of child social phobia symptoms, administered before and after treatment for social phobia. Participants included a clinic sample of 81 children (7-16 years old $[\mathrm{M}=11.75, \mathrm{SD}=2.57] ; 39$ girls, 42 boys) and their parents receiving treatment as part of a multisite controlled trial. Pretreatment parent-child reporting discrepancies predicted
\end{abstract}

This research was supported in part by NIMH grant R01MH53703 to the third author. Lilly Corporation supplied the fluoxetine and matching placebo capsules. Clinical trial registration informationURL: http://www.clinicaltrials.gov; Unique identifier: NCT00043537.

A. De Los Reyes $(\bowtie)$

Department of Psychology,

University of Maryland at College Park,

Biology/Psychology Building, Room 3123H,

College Park, MD 20742, USA

e-mail: adelosreyes@psyc.umd.edu

\section{A. Alfano}

Children's National Medical Center,

The George Washington School of Medicine,

111 Michigan Avenue, NW,

Washington DC 20010, USA

e-mail: calfano@cnmc.org

\section{C. Beidel}

Department of Psychology, University of Central Florida,

4000 Central Florida Blvd,

Orlando, FL 32816, USA

e-mail: dbeidel@mail.ucf.edu parent-child discrepancies at posttreatment, and these relations were not better accounted for by the severity of the child's pretreatment primary diagnosis. Further, treatment responder status moderated this relation: Significant relations were identified for treatment non-responders and not for treatment responders. Overall, findings suggest that informant discrepancies can be reliably employed to measure individual differences over the course of controlled treatment trials. These data provide additional empirical support for recent work suggesting that informant discrepancies can meaningfully inform understanding of treatment response as well as variability in treatment outcomes.

Keywords Attribution Bias Context Model · Efficacy · Informant discrepancies · Intervention ·

Range of Possible Changes Model

The absence of definitive measures for assessing clinical conditions (e.g., anxiety, depression, disruptive behavior) in children and adolescents (hereafter referred to collectively as "children") makes it critical to assess these conditions from the perspectives of multiple informants (e.g., self, significant other, clinician, laboratory observer, biological indices). However, multiple clinical reports of a single child are often in disagreement, both in terms of the level or severity of symptoms and whether a child meets diagnostic criteria (De Los Reyes and Kazdin 2005). These disagreements (hereafter referred to as "informant discrepancies") are some of the most consistent effects observed in the clinical child literature (Achenbach 2006).

Informant discrepancies are critical to understand for numerous reasons. First, they commonly occur across measurement methods (De Los Reyes and Kazdin 2005) 
and areas of the clinical sciences (e.g., Achenbach et al. 1987; Clancy et al. 2005; De Los Reyes and Prinstein 2004), suggesting that the phenomenon is of general concern to researchers interested in studying children's behavior. Further, prior work suggests that differences in the magnitudes of informant discrepancies are dependent upon the problem type (e.g., internalizing versus externalizing concerns) as well as the informant pair (e.g., parent-child, mother-father, parent-teacher) (Achenbach 2006). However, most discrepancies typically fall in the moderate-to-large range (De Los Reyes and Kazdin 2005). Second, informant discrepancies frequently translate into inconsistent outcomes within controlled treatment trials (e.g., De Los Reyes and Kazdin 2008; Weisz et al. 2006; Weisz et al. 1987), making it difficult to definitively determine treatment efficacy. Third, discrepancies may indicate the development of poor child and family psychosocial outcomes. For instance, when a parent and child disagree in their reports of important behaviors (e.g., the child's behavior and emotional problems, parenting styles, and parent-child relationship quality), these disagreements predict negative outcomes including poorer treatment response, poor parental participation in the child's therapy, and increases in the child's behavioral and emotional problems (Ferdinand et al. 2004; Guion et al. 2009; Israel et al. 2007; Panichelli-Mindel et al. 2005; Pelton and Forehand 2001; Pelton et al. 2001). In short, informant discrepancies occur often and have numerous implications for the assessment, development, and treatment of child psychopathology.

Recently, researchers have focused on whether informant discrepancies may aid in understanding the outcomes of controlled treatment trials (De Los Reyes and Kazdin 2006a, 2009; Koenig et al. 2009). Indeed, when outcome reports are inconsistent, one might initially question whether treatment yielded positive outcomes and/or whether some of the informants' reports were unreliable. Alternatively, discrepancies might reveal the circumstances in which a treatment is effective (i.e., mediators and moderators of outcome). For example, parent reports might suggest that the treatment was effective whereas teacher reports might suggest a lack of improvement. These differential outcomes might indicate that: (a) the intervention significantly reduced problematic behaviors expressed in the home (e.g., negative interactions with siblings) but not in non-home settings; and/or (b) significant changes in behavior occurred at school but teacher reports were unreliable or did not accurately represent these changes. Interestingly, examining whether discrepancies yield meaningful information is a testable question rarely addressed in the clinical literature. Yet, examining these issues may yield knowledge on how outcomes vary for different children.

Recent research highlights the utility of informant discrepancies for informing controlled trials research. First, meta-analytic evidence suggests that the multiple outcome measures commonly used to test treatment efficacy can be examined to identify patterns of consistent and inconsistent treatment effects (De Los Reyes and Kazdin 2009). For example, behavioral parent training interventions developed to treat childhood disruptive behavior commonly use outcomes measures administered to parents, teachers, and laboratory observers. Yet, robust informant-specific effects (i.e., multiple measures completed by the same informant suggesting positive treatment outcomes) are most often observed via parent-reported outcomes (De Los Reyes and Kazdin 2009). On the surface, such findings might suggest that reports provided by informants other than parents are unreliable or that treatment was ineffective because parent reports were not corroborated by other informants' reports. However, recent work calls these interpretations into question. For instance, parent-teacher discrepancies in reports of preschoolers' disruptive behavior symptoms predict laboratory observations of the interactions in which these same preschoolers exhibit disruptive behavior (De Los Reyes et al. 2009a). Specifically: (a) parent reports (and not teacher reports) predict observed disruptive behavior within parent-child interactions; (b) teacher reports (and not parent reports) predict observed disruptive behavior within interactions between the child and a nonparental adult (clinical examiner); and (c) when combined, parent and teacher reports predict disruptive behavior that is observed across adult-child interactions. Taken together, these findings suggest a systematic nature to informant discrepancies; so much so that within controlled treatment trials, discrepancies may reveal where (e.g., home, school) a treatment changed children's behavior.

Informant discrepancies may contain meaningful information, leading to hypotheses as to how and why treatments change behavior within controlled trials. However, the field has a poor understanding of the psychometric properties of informant discrepancies. It remains unclear whether measures of discrepancies have the same psychometric properties as reliable and valid assessment measures. For instance, do repeated measurements of informant discrepancies relate to each other, such that informant discrepancies observed at one time point (e.g., prior to treatment) predict discrepancies at other time points (e.g., once treatment is complete)? Indeed, understanding the stability of informant discrepancies is necessary to clarify treatment response; understanding this stability would allow researchers to predict the presence of informant discrepancies at multiple points (e.g., baseline, posttreatment, follow-up).

Prior theoretical work suggests that informant discrepancies may indeed exhibit stable measurement properties. The Attribution Bias Context Model (De Los Reyes and Kazdin 2005) is a multidisciplinary framework that attempts to explain why informant discrepancies exist in clinical child 
assessments. The framework builds on basic research and theory on memory and interpersonal perception as well as multi-informant clinical assessment (Achenbach 2006; Johnson et al. 1993; Jones and Nisbett 1972; Malle 2006; Mash and Terdal 1988; Pasupathi 2001). Briefly, the framework posits that discrepant reports of a given behavior exist because informants differ on three critical characteristics: (a) what they attribute to be the causes of the behavior (i.e., dispositional qualities of the child versus environmental constraints); (b) the perspectives by which they perceive whether or which behaviors warrant treatment; and (c) the contexts within which they observe the behavior. Thus, informant discrepancies occur because of systematic differences between informants' experiences with and perceptions of the behavior being assessed. As such, these systematic differences should translate into predictable differences between informants' reports over multiple assessments.

The basic tenets of the Attribution Bias Context Model have empirical support. For example, as mentioned previously informant discrepancies may be useful markers of the variable expression of children's behavior across settings (De Los Reyes et al. 2009a). However, whether informant discrepancies reflect systematic variations between informants' reports over time has not been empirically tested. Therefore, the present study examines whether informant discrepancies assessed before children's treatments predict such discrepancies after treatment is complete, using a sample of children treated for social phobia.

There are four key reasons for why informant discrepancies are crucial to study within controlled trials testing treatments, including childhood social phobia. First, observed high rates of reporting disagreements within clinic samples of anxious children are commonly reported (Choudhury et al. 2003; Grills and Ollendick 2003; Rapee et al. 1994). Second, controlled treatment trials for childhood anxiety commonly use outcome measures from multiple informants (e.g., Beidel et al. 2007; Kendall 1994; Silverman et al., 1999a, b; Weisz et al. 2005). Third, informant disagreements occur at pretreatment, posttreatment, and longitudinal follow-up (Safford et al. 2005). This suggests that variability exists between informants' reports across multiple time points. Thus, there is potential for pretreatment discrepancies to predict discrepancies over time.

Fourth and most critically, recent work suggests that systematic patterns exist in parental and child reports of clinic referred children's anxiety symptoms. Specifically, when parents and children endorse symptoms during structured diagnostic interviews, disagreements tend to be lower when the symptoms assessed are observable (e.g., behavioral avoidance) and based on behaviors exhibited in non-school settings. In contrast, higher disagreements occur when the symptoms assessed are unobservable or subjectively expressed (e.g., anxious worry) and based on behaviors exhibited in school settings (Comer and Kendall 2004). Thus, akin to parent and teacher reports of disruptive behavior (De Los Reyes et al. 2009a), parent and child reports of anxiety symptoms vary systematically in terms of the nature and setting of the child's symptoms. However, studies of measurements of informant discrepancies and whether they systematically relate to each other are lacking in empirical work on clinically anxious children.

Conceivably, several factors may moderate the relation between discrepancies measurements. One factor particularly relevant to controlled trials is the child's treatment response. Specifically, independent observers (e.g., clinician, laboratory observer) often determine whether a child experiences positive treatment response based on interviews with other informants (e.g., parent, child, and/or teacher; Guy 1976; Shaffer et al. 1983; Silverman and Albano 1996). If identified as a treatment responder, this might signify that informants agreed that the treatment improved the child's functioning. This is the independent evaluator's judgment, which relies on information from informants. Thus, one might assume that all informants provided consistent perceptions of improvement. Yet, consistent perceptions of improvement at posttreatment may prevent informant discrepancies from demonstrating stability over the course of treatment. This is because at the end of treatment, all informants likely see the child's response to treatment in much the same way.

Conversely, when a child is not identified as a treatment responder, informants may disagree that the child responded to treatment. As a result, the informant discrepancies observed at posttreatment for non-responding children likely resemble the informant discrepancies observed at pretreatment. Because informant discrepancies are consistently observed in the absence of treatment (De Los Reyes and Kazdin 2005), treatment non-response may reveal the considerable predictive utility of pretreatment informant discrepancies. Thus, a second aim of the present study is to examine whether the predictive utility of pretreatment parentchild reporting discrepancies is moderated by whether the child is identified by an independent evaluator as responding positively to treatment.

This study examined whether pretreatment informant discrepancies in reports of child social phobia predict posttreatment informant discrepancies. We addressed this question using data from a multisite controlled clinical trial of psychological and pharmacological treatments for childhood social phobia (Beidel et al. 2007). On the assumption that we would identify the low-to-moderate levels of agreement commonly observed in prior work (De Los Reyes and Kazdin 2005), we predicted that pretreatment parent-child reporting discrepancies would predict posttreatment discrepancies. We expected that this rela- 
tionship would be robust to taking into account pretreatment diagnostic severity. This is important because clinicians often rely on parent and child reports to determine a child's baseline level of functioning (Hawley and Weisz 2003). Additionally, we expected that the relation between pretreatment and posttreatment informant discrepancies would be moderated by child responder status at posttreatment. That is, a predictive effect would be present for treatment non-responders but not for treatment responders.

\section{Method}

\section{Participants}

Participants included 81 parent-child (7-16 years old [M= $11.75, \mathrm{SD}=2.57$ ]; 39 girls, 42 boys) dyads for which the child was receiving treatment as part of a multisite controlled trial comparing pharmacological (fluoxetine) and behavioral (Social Effectiveness Therapy for Children [SET-C]; Beidel et al. 2000) treatments for social phobia (see Beidel et al. 2007). Recruitment occurred through media announcements (newspaper, radio) or through referrals from local mental health professionals. All children met criteria for a primary diagnosis of social phobia, generalized subtype (American Psychiatric Association 2000) based on child and parent semi-structured diagnostic interviews (Anxiety Disorders Interview Schedule for Children [ADIS-C/P]; Silverman and Albano 1996). In the case of parent-child reporting discrepancies, clinicians arrived at a diagnosis through composite diagnostic procedures for integrating parent and child reports as outlined by Silverman and Albano (1996).

To be included in the controlled trial, youths had to have a primary diagnosis of social phobia. To be considered primary, social phobia symptoms were of at least moderate severity (4 or higher on an 8-point scale) and created functional impairment. Coexisting disorders could not have higher severity ratings or cause more substantial impairment. To ensure generalization of study findings, secondary comorbid diagnoses were allowed, with the exception of bipolar disorder, psychosis, conduct disorder, autism spectrum disorders, and mental retardation. Youth with moderate to severe depression who expressed active suicidal ideation or who had a previous unsuccessful trial of fluoxetine or behavior therapy were excluded. In the present sample, parents and children were included if they provided complete questionnaire information on the child's social phobia symptoms at pretreatment and immediately posttreatment. Thus, this sample was culled from a larger sample of 139 dyads. Detailed information on demographic and clinical characteristics for the total sample has been reported elsewhere (Beidel et al. 2007). ${ }^{1}$

\section{Measures}

Social phobia symptoms Both parents and children completed respective versions of the Social Phobia and Anxiety Inventory for Children (SPAIC; Beidel et al. 1995) at pre- and posttreatment. The scale consists of 26 items that assess the range of situations known to be anxiety provoking to children with social phobia. We used the SPAIC total score for which acceptable levels of internal consistency and test-retest reliability have been reported and for which well established psychometric properties for assessing clinically relevant social phobia in children are available (Silverman and Ollendick 2005). At pretreatment, Cronbach's alpha coefficients for this sample were 0.95 for the parent-report items and 0.97 for the child-report items (alpha analyses based on complete item data for 77 parents and 79 children). At posttreatment, Cronbach's alpha coefficients for this sample were 0.96 for the parent-report items and 0.97 for the child-report items (alpha analyses based on complete item data for 75 parents and 78 children).

Reporting discrepancies on childhood social phobia symptoms Parent-child reporting discrepancies were assessed using standardized difference scores (SDS), consistent with current recommendations and practices (e.g., De Los Reyes et al. 2008; De Los Reyes and Kazdin 2004, 2006b; Guion et al. 2009; Owens et al. 2007; Weems et al. 2007; Weems et al., in press). Standardized difference scores were created by first converting each SPAIC total score into a $z$ score and then subtracting the child's SPAIC $z$ score from the parent's SPAIC $z$ score (hereby referred to as SPAIC-Standardized Difference Scores [SPAIC-SDS]). Alternative measurements of informant discrepancies have been proposed and used in prior work, such as raw difference scores (i.e., use of

\footnotetext{
${ }^{1}$ We conducted exploratory analyses to examine whether the 81 parents and children examined in this study differed from the rest of the sample at pretreatment as a function of demographic (e.g., child age, gender, ethnicity, socioeconomic status), pretreatment functioning (e.g., primary diagnosis type, clinical severity of primary diagnosis, broadband internalizing and externalizing problems [Child Behavior Checklist; Achenbach 1991], SPAIC scores), or treatment characteristics (e.g., treatment condition, treatment site). We conducted a large number of tests $(n=13)$ and did not have a priori hypotheses to advance. Thus, we set a pre-defined bonferroni-corrected $p$-value threshold for these tests of 0.004 (i.e., .05/13). Across these 13 tests, none of these factors evidenced a significant relation to inclusion/ exclusion into this study.
} 
unstandardized rather than standardized scores) and residual difference scores (i.e., measurements of the unshared variance between two informants' scores). However, standardized difference scores are a better representation of informant discrepancies, because reports from which discrepancies scores are created often correlate too highly with raw difference and residual difference scores (see De Los Reyes and Kazdin 2004). That is, raw difference and residual difference scores are often statistically redundant with the informants' ratings used to compute the scores. Further discussions of the mathematical properties of SDS can be found elsewhere (De Los Reyes and Kazdin 2004; Guion et al. 2009; Owens et al. 2007).

Clinical severity of primary diagnosis Interviews of the parent and child were conducted using the ADIS-C/P (Silverman and Albano 1996). Interviewers assigned a clinical severity rating for each disorder present using the 8point Clinical Severity Rating Scale (ADIS-C/P CSR). For the total sample, $20 \%$ of the interviews were audiotaped and scored by a second interviewer $(10 \%$ of the tapes from one site were sent to the other site to ensure intersite reliability). For the total sample, we observed strong interrater reliability for a social phobia diagnosis (kappa= 0.78). Other diagnoses were not diagnosed with sufficient frequency to allow ratings of interrater agreement. For the total sample, interrater reliability for the social phobia CSR was $r=0.82$.

Treatment responder status Treatment responder status was assessed using the Clinical Global Impressions (CGI) Severity of Illness and Improvement Scale (Guy 1976). The CGI was completed by the pretreatment diagnostic interviewer and by a posttreatment independent evaluator blinded to group status (12 weeks). The interrater reliability for the CGI, conducted for $20 \%$ of the total sample, was $r=0.86$. A priori and consistent with other pharmacological trials (Birmaher et al. 2003; Wagner et al. 2004), ratings of 1 (very much improved) or 2 (much improved) on the CGI denoted a treatment responder, with ratings based on the evaluator's separate interviews with parent and child. Thus, treatment responder status was dummy-coded " 0 " (not a treatment responder) or "1" (treatment responder) (see Table 1 for percentages).

\section{Procedure}

Parents contacted the clinic in response to study advertisements or referrals from mental health professionals. Parents received a scripted explanation of the study and participated in a telephone interview, conducted by doctoral level graduate students or trained staff members. The telephone interview was used to screen for symptoms of social phobia and other Axis I disorders, as well as the child's psychological and medical history. If the child met the basic eligibility requirements, the family was invited to the clinic to complete the ADIS-C/P and a battery of questionnaires.

During the initial study visit, parent and child participated in a diagnostic interview conducted by a psychiatrist, clinical psychologist, or doctoral student in clinical psychology using the ADIS-C/P (Silverman and Albano 1996). Parents were interviewed first, then the same clinician interviewed the child. Diagnoses were based on information provided by both informants. In the case of parent-child disagreements, the final diagnosis was derived using composite diagnostic procedures for integrating parent and child reports as outlined by Silverman and Albano (1996). The diagnostic interview was repeated at posttreatment using the same procedure. Further information on study procedures, treatments, and inclusion criteria have been reported elsewhere (Beidel et al. 2007).

\section{Results}

\section{Preliminary Analyses}

Frequency distributions examined for all variables did not reveal any deviations from normality. Correlations computed between child and parent total SPAIC scores at pretreatment $(r=0.49, p<001)$ and posttreatment $(r=0.30, p<0.01)$ revealed significant but low-to-moderate correspondence between parent and child ratings, consistent with previous investigations (Achenbach 2006; De Los Reyes and Kazdin 2005). ${ }^{2}$

Means, standard deviations, and correlations among measures Means and standard deviations (and proportions for treatment responder status) for the measures completed

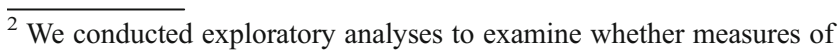
parent-child reporting discrepancies at either pre- or posttreatment varied as a function of demographic (e.g., child age, gender, ethnicity, socioeconomic status), pretreatment functioning (e.g., primary diagnosis type, clinical severity of primary diagnosis, broadband internalizing and externalizing problems [Child Behavior Checklist; Achenbach 1991]), or treatment characteristics (e.g., treatment condition, treatment site, treatment attrition, treatment response status). We conducted a large number of tests $(n=30)$ and did not have a priori hypotheses to advance. Thus, we set a pre-defined bonferroni-corrected $p$-value threshold of 0.001 (i.e., .05/30). Across these 30 tests, none of these factors had a significant relationship to either pre- or posttreatment reporting discrepancies.
} 
Table 1 Means (M) and

Standard Deviations (SD) of

Measures for the Total

Sample $(N=81)$

SPAIC Social Phobia and Anxiety Inventory for Children; $A D I S-C / P$ Anxiety Disorders Interview Schedule for Children; CSR Clinician Severity Rating

\begin{tabular}{|c|c|c|c|c|c|c|}
\hline \multirow[t]{2}{*}{ Measure } & \multicolumn{2}{|l|}{ Parent } & \multicolumn{2}{|l|}{ Child } & \multicolumn{2}{|c|}{ Clinician } \\
\hline & $M$ & $(S D)$ & $M$ & $(S D)$ & $M$ & $(S D)$ \\
\hline \multicolumn{7}{|l|}{ 1. SPAIC Total Score } \\
\hline a. Pretreatment & 34.57 & $(8.50)$ & 27.75 & $(8.89)$ & & \\
\hline b. Posttreatment & 24.69 & $(10.43)$ & 16.49 & $(10.84)$ & & \\
\hline \multicolumn{7}{|l|}{ ADIS-C/P Primary Diagnosis CSR, } \\
\hline Pretreatment & & & & & 5.98 & $(1.00)$ \\
\hline Treatment Responder Status (\%) & & & & & 32 & \\
\hline
\end{tabular}

by parent, child, and clinician are presented in Table 1. Correlations were calculated between continuous variables used in the main tests of our hypotheses and SPAIC-SDS (see Table 2). We identified significant bivariate relations between pre- and posttreatment SPAIC-SDS. However, SPAIC-SDS were not related to pretreatment ADIS-C/P CSR. This is consistent with prior work suggesting that informant discrepancies are not related to clinical impairment (De Los Reyes et al. 2009a; De Los Reyes et al. 2009b). Nevertheless, we controlled for pretreatment CSR in regression analyses in order to provide more conservative tests of our hypotheses.

Pre-Treatment Parent-Child Discrepancies among Treatment Responders and Non-Responders

To first examine whether the two treatment response groups differed in terms of pretreatment parent-child discrepancies, we compared treatment responders and non-responders via $t$-test group comparisons on pretreatment SPAIC-SDS. We identified non-significant differences between treatment non-responders, $n=55 ; M=-0.08 ; S D=0.98$, and treatment responders, $n=26 ; M=0.18 ; S D=1.06$, on pretreatment SPAIC-SDS, $t=-1.11, n s$. This suggests that the predictive findings we report below are not simply due to pretreatment differences between treatment responders and nonresponders on parent-child reporting discrepancies.

Table 2 Correlations among Pre- and Posttreatment Parent-Child Reporting Discrepancies (i.e., Standardized Difference Scores) on Social Phobia Symptoms and Pretreatment Clinical Severity for the Total Sample $(N=81)$

\begin{tabular}{llll}
\hline Variable & 1 & 2 & 3 \\
\hline 1. SPAIC-SDS, Pretreatment & & $0.24^{*}$ & -0.01 \\
2. SPAIC-SDS, Posttreatment & & -0.19 \\
3. ADIS-C/P CSR, Pretreatment & & \\
\hline
\end{tabular}

SPAIC Social Phobia and Anxiety Inventory for Children; SDS Standardized Difference Score; $A D I S-C / P$ Anxiety Disorders Interview Schedule for Children; CSR Clinician Severity Rating; ${ }^{*} p<0.05$
Predicting Posttreatment Parent-Child Discrepancies from Pretreatment Discrepancies

To examine whether pretreatment parent-child discrepancies of social phobia symptoms predict posttreatment parent-child discrepancies after controlling for pretreatment clinical severity, we conducted a hierarchical regression analysis with posttreatment SPAIC-SDS serving as the criterion variable. We entered pretreatment ADIS-C/P CSR (continuous) in the first step as an independent variable. In the second step, we entered as independent variables pretreatment SPAIC-SDS (continuous), treatment responder status (dichotomous), and the interaction term for these two variables. In the presence of a significant interaction effect, we used Holmbeck's (2002) guidelines for post-hoc probing of significant moderator effects. This included: (a) computation of slope estimates using centered variables (reducing multicollinearity) and (b) examining the statistical significance of these slopes for each level of treatment responder status (i.e., the moderator variable). Results are presented in Table 3.

Main regression model Results were consistent with our hypotheses (Table 3). Specifically, in the first step of the equation pretreatment ADIS-C/P CSR were not significantly related to posttreatment SPAIC-SDS. In the second step, the addition of pretreatment SPAIC-SDS, treatment responder status, and their interaction contributed significant variance to the regression model. Specifically, after controlling for pretreatment ADIS-C/P CSR, pretreatment SPAIC-SDS significantly predicted posttreatment SPAICSDS. Although treatment responder status did not independently account for significant variance in the model, the interaction between pretreatment SPAIC-SDS and treatment responder status predicted posttreatment SPAIC-SDS.

Post-hoc probing of significant interaction Results of posthoc tests revealed a positive, significant slope between preand posttreatment SPAIC-SDS for treatment non-responders. Specifically, for non-responders greater levels of pretreatment SPAIC-SDS predicted greater levels of posttreatment SPAICSDS. The magnitude of this finding approached a large effect 
Table 3 Hierarchical Regression Analyses Examining Pretreatment Parent-Child Reporting Discrepancies (i.e., Standardized Difference Scores) on Social Phobia Symptoms, Treatment Responder Status, and
Their Interaction in Predicting Posttreatment Parent-Child Reporting Discrepancies on Social Phobia Symptoms

\begin{tabular}{|c|c|c|c|c|c|c|c|c|c|}
\hline \multirow[t]{2}{*}{ Variable } & \multicolumn{4}{|c|}{ Main regression model } & \multirow[t]{2}{*}{ Variable } & \multicolumn{4}{|c|}{ Post-Hoc tests of moderation } \\
\hline & $\Delta \mathrm{R}^{2}$ & $\mathrm{~B}$ & $\mathrm{SeB}$ & $\beta$ & & $\Delta \mathrm{R}^{2}$ & $\mathrm{~B}$ & $\mathrm{SeB}$ & $\beta$ \\
\hline Step 1 & 0.03 & & & & Step $2($ When No Response $=0)$ & $0.15^{*}$ & & & \\
\hline \multirow[t]{3}{*}{ ADIS-C/P CSR, Pretreatment } & & -0.21 & 0.12 & -0.18 & SPAIC-SDS & & 0.54 & 0.15 & $0.47^{*}$ \\
\hline & & & & & Treatment Responder Status & & -0.25 & 0.26 & -0.10 \\
\hline & & & & & SDS X Responder Interaction & & -0.70 & 0.25 & $-0.35^{*}$ \\
\hline Step 2 & $0.15^{*}$ & & & & Step $2($ When Response $=0)$ & $0.15^{*}$ & & & \\
\hline SPAIC-SDS & & 0.54 & 0.15 & $0.47^{*}$ & SPAIC-SDS & & -0.15 & 0.20 & -0.13 \\
\hline Treatment Responder Status & & -0.25 & 0.26 & -0.10 & Treatment Responder Status & & -0.25 & 0.26 & -0.10 \\
\hline SDS X Responder Interaction & & -0.70 & 0.25 & $-0.35^{*}$ & SDS X Responder Interaction & & -0.70 & 0.25 & $-0.48^{*}$ \\
\hline
\end{tabular}

For the main regression model (left side of table), Treatment Responder Status is coded " 0 " for No Response and " 1 " for Response; regression terms for variables entered at Steps 1 and 2 are displayed, based on terms observed for these variables in Step 2 of the model; $\Delta \mathrm{R}^{2}$ statistics for each step were based on variables entered in that step; for the post-hoc moderation tests (right side of table), only Step 2 is reported (pretreatment clinical severity was again a non-significant predictor in the first step), with the moderator variable Treatment Responder Status manipulated to reflect when the absence of treatment response is coded 0 ("When No Response $=0$ "), and when treatment response is coded 0 ("When Response $=$ 0") (see Holmbeck 2002); SPAIC Social Phobia and Anxiety Inventory for Children; SDS Standardized Difference Score; ADIS-C/P Anxiety Disorders Interview Schedule for Children; CSR Clinician Severity Rating; ${ }^{*} p<0.01$

$(r=0.50)$ as delineated by Cohen's (1988) effect size conventions, $\beta=0.47 ; t=3.63, p<0.01$. By comparison, we observed a non-significant slope for treatment responders, $\beta=-0.13 ; t=-0.75, n s$.

\section{Discussion}

\section{Main Findings}

Despite consistently high rates of informant discrepancies in clinical child assessments, little is known about the stability and utility of these commonly observed phenomena, particularly within controlled trials research. This study sheds significant light on the issue of informant discrepancies and the interpretation of treatment outcome data. Within a multisite controlled treatment trial for childhood social phobia, we found that initial parent-child reporting discrepancies predicted discrepancies over time, and this prediction was sufficiently robust to account for the severity of pretreatment primary diagnosis. Further, this prediction was moderated by treatment response. Specifically, we found a significant and large predictive effect of pretreatment discrepancies on posttreatment discrepancies and only for children identified as treatment nonresponders. This finding is particularly interesting in light of the fact that treatment responders and non-responders did not significantly differ in their degree of pretreatment parent-child reporting discrepancies.

The findings highlight the systematic nature of informant discrepancies. We identified predictive effects where one might expect to find differences between parent and child perceptions of treatment outcome. Indeed, in this study an independent evaluator blind to treatment condition interviewed the parent and child (separately) in order to assess the child's improvement at posttreatment. As such, when independent evaluators identified treatment non-responders, this might characterize parents and children who discrepantly viewed improvements in the child's functioning. Thus, one would expect pretreatment discrepancies to predict posttreatment discrepancies, because treatment likely did not change parent and child views of behavior and the child's improvement over the course of treatment. That is, parent and child were quite likely to discrepantly perceive the child's problems at outcome in much the same way as they discrepantly perceived the child's problems before treatment began.

In contrast, when independent evaluators identified treatment responders, this might characterize circumstances where parents and children were likely to agree that treatment improved the child's functioning. For children identified as treatment responders, this likely was in stark contrast to how parents and children viewed the child's functioning before treatment began. In fact, numerous studies suggest that in the absence of treatment, parent and child often disagree on the nature and extent of the child's problems (Achenbach 2006). Here, the ability of pretreatment informant discrepancies to predict posttreatment discrepancies would not be expected, because parent and child likely largely perceived the child's target problems discrepantly at baseline but largely agreed in their perceptions of the child's target problems at treatment 
outcome. Stated another way, unlike treatment nonresponders, treatment responders were characterized by significant improvements at post-treatment compared to their functioning at baseline. This change likely prevented parent-child discrepancies from demonstrating stability over the course of treatment. Therefore, the findings suggest that systematic and conceptually meaningful relations exist between pre- and posttreatment informant discrepancies and the extent of the child's response to treatment.

\section{Limitations}

There are limitations to the present study. First, informant discrepancies were assessed using standardized difference scores. Some researchers have raised concerns about the reliability of difference scores for assessing constructs such as informant discrepancies and general variations between scores (e.g., De Los Reyes and Kazdin 2004; Nunnally and Bernstein 1994; Rogosa et al. 1982; Rogosa and Willett 1983). However, prior work has noted that when differences between informants' reports exist, difference scores demonstrate acceptable levels of reliability (Rogosa et al. 1982; Rogosa and Willett 1983). In any event, future research should use different approaches to assess discrepancies (e.g., direct assessments of perceived discrepancies).

Second, we did not assess parent and child perceptions of treatment response independently of independent evaluator ratings. As such, we were unable to test for differences between independent evaluator-identified treatment responders and non-responders and parent and child perceptions of posttreatment change. We encourage future research to replicate and extend our findings using multiple informants' reports of treatment response.

Third, we did not account for other factors that might have reduced the stability between pre- and posttreatment discrepancies. For example, we did not assess the degree to which parent and child reports focused on the same social phobia problems and behaviors at pre- and posttreatment (i.e., response shift bias; see Kazdin 2003). For instance, some children's reports may have been more focused on behaviors/concerns in one domain/setting before treatment but "switched" in focus by posttreatment (e.g., school anxiety at pretreatment but sports-related anxiety at posttreatment). If parents and children (a) do not evidence the same kind of response shift or (b) are discordant in whether they engaged in response shift, this may promote instability in discrepant reports over time. Thus, it may be that our findings underestimate the stability of informant discrepancies within controlled trials. These issues can be addressed in future research. For instance, after completing their pretreatment reports, researchers may solicit information from informants on the behaviors (behavioral avoidance, physiological arousal) or circumstances (home, school) upon which they tended to base their reports. At the end of treatment, informants can be randomly assigned to either complete the measures in their standard format or receive instructions on how to base their reports on the same behaviors or circumstances upon which they based their pretreatment reports. In any event, future research ought to examine whether correcting for response shift increases the predictive utility of pretreatment informant discrepancies.

Lastly, parent-child discrepancies were assessed with the same measures at pre- and posttreatment. In many respects this is a necessary criterion for conducting research on informant discrepancies in that it ensures that assessments are not confounded by differences in item content across reports (see De Los Reyes and Kazdin 2004; Treutler and Epkins 2003). At the same time, this may have inflated the obtained relationships. Shared method variance due to the common assessment formats and informants could have increased correlations among measures. Of course, this would happen for control variables (e.g., clinical severity ratings, which are based on clinician, parent, and child report), independent variables (e.g., pretreatment discrepancy scores, treatment responder status, the interaction between these two variables), and dependent variables (e.g., posttreatment discrepancy scores). That is, common method variance could increase the likelihood that non-significant findings would emerge, because the grand majority of study variables were derived from highly similar methods, and variables were often derived from information derived from the same informants. Nevertheless, future research should consider additional measures, including ratings from other informants (e.g., fathers, teachers) and measures in the home or other settings (e.g., school) that do not rely on the same informants' reports.

\section{Research and Clinical Implications}

As mentioned previously, informant discrepancies yield meaningful information on the circumstances or contexts within which children exhibit problematic behavior (De Los Reyes and Kazdin 2006a, 2008, 2009; De Los Reyes et al. 2009a). In line with prior work, the findings suggest consistency in informant discrepancies as observed within controlled trials. This suggests that informant discrepancies might be used as individual differences variables. Examining individual differences in informant discrepancies may lead to a better understanding of children's treatment responsiveness. Indeed, informant discrepancies do not necessarily imply that one or both of the informants' reports are unreliable or that treatment was ineffective. Consistencies in informant discrepancies over the course of treatment might point to circumstances within which children experience improvement versus those within which concerns remain. Using traditional methods such as 
clinical ratings of improvement (e.g., CGI; Guy 1976), it may be that informants often have to agree that the child's functioning improved in order for an independent evaluator to identify the child as a treatment responder. Thus, when informant discrepancies are consistently observed over the course of treatment, traditional methods may not be sensitive enough to identify how or under which circumstances improvement occurred. Alternatively, consistent informant discrepancies over the course of treatment may indicate that children simply have not responded to treatment. These issues are worthy of empirical study to establish whether consistently observed informant discrepancies suggest a failure in treatment response or positive responses observed under some circumstances and not others.

Our findings also have important implications for clinical practice. Indeed, practitioners often observe the same kinds of informant discrepancies in non-laboratory based settings (e.g., Hawley and Weisz 2003). In fact, it is highly likely that when a practitioner administers similar measures to more than one informant during an intake, informant discrepancies will arise. Our findings suggest that clinicians have an opportunity to use these discrepancies to guide assessment and treatment. Specifically, after intake, each informant might be asked (independently) where they typically observe the child's problematic behaviors. At the end of treatment, if informants disagree about treatment effectiveness, a clinician could query specifically regarding the situations where problems evident before treatment no longer exist, as well as the situations where behavioral problems still exist. Based on this information, discrepancies could generate hypotheses about variable outcomes, and guide clinicians to modify treatment plans to address outstanding concerns. In sum, we encourage researchers and practitioners to use informant discrepancies to guide hypothesis generation and testing to understand children's responses to treatment and variability in these responses.

Acknowledgement The authors acknowledge the efforts of Patricia Rao, Ph.D., Project Coordinator, Richard Gross, M.D., and Stephen Kwass, M.D., psychiatrists.

\section{References}

Achenbach, T. M. (1991). Manual for the Child Behavior Checklist 14-18 and 1991 profile. Burlington: University of Vermont.

Achenbach, T. M. (2006). As others see us: Clinical and research implications of cross-informant correlations for psychopathology. Current Directions in Psychological Science, 15, 94-98.

Achenbach, T. M., McConaughy, S. H., \& Howell, C. T. (1987). Child/adolescent behavioral and emotional problems: Implications of cross-informant correlations for situational specificity. Psychological Bulletin, 101, 213-232.

American Psychiatric Association. (2000). Diagnostic and statistical manual of mental disorders (4th ed.), text revision (DSM-IV-TR). Washington, DC: Author.
Beidel, D. C., Turner, S. M., \& Morris, T. L. (1995). A new inventory to assess childhood social anxiety and phobia: The Social Phobia and Anxiety Inventory for Children. Psychological Assessment, 7, 73-79.

Beidel, D. C., Turner, S. M., \& Morris, T. L. (2000). Behavioral treatment of childhood social phobia. Journal of Consulting and Clinical Psychology, 68, 1072-1080.

Beidel, D. C., Turner, S. M., Sallee, F. R., Ammerman, R. T., Crosby, L. A., \& Pathak, S. (2007). SET-C versus fluoxetine in the treatment of childhood social phobia. Journal of the American Academy of Child and Adolescent Psychiatry, 46, 1622-1632.

Birmaher, B., Axelson, D. A., Monk, K., Kalas, C., Clark, D. B., Ehmann, M., et al. (2003). Fluoxetine for the treatment of childhood anxiety disorders. Journal of the American Academy of Child and Adolescent Psychiatry, 42, 415-423.

Choudhury, M. S., Pimentel, S. S., \& Kendall, P. C. (2003). Childhood anxiety disorders: Parent- child (dis)agreement using a structured interview for the DSM-IV. Journal of the American Academy of Child \& Adolescent Psychiatry, 42, 957-964.

Clancy, C. A., McGrath, P. J., \& Oddson, B. E. (2005). Pain in children and adolescents with spina bifida. Developmental Medicine and Child Neurology, 47, 27-34.

Comer, J. S., \& Kendall, P. C. (2004). A symptom-level examination of parent-child agreement in the diagnosis of anxious youth. Journal of the American Academy of Child and Adolescent Psychiatry, 43, 878-886.

De Los Reyes, A., \& Kazdin, A. E. (2004). Measuring informant discrepancies in clinical child research. Psychological Assessment, 16, 330-334.

De Los Reyes, A., \& Kazdin, A. E. (2005). Informant discrepancies in the assessment of childhood psychopathology: A critical review, theoretical framework, and recommendations for further study. Psychological Bulletin, 131, 483-509.

De Los Reyes, A., \& Kazdin, A. E. (2006a). Conceptualizing changes in behavior in intervention research: The range of possible changes model. Psychological Review, 113, 554-583.

De Los Reyes, A., \& Kazdin, A. E. (2006b). Informant discrepancies in assessing child dysfunction relate to dysfunction within mother-child interactions. Journal of Child and Family Studies, $15,643-661$.

De Los Reyes, A., \& Kazdin, A. E. (2008). When the evidence says, "Yes, no, and maybe so": Attending to and interpreting inconsistent findings among evidence-based interventions. Current Directions in Psychological Science, 17, 47-51.

De Los Reyes, A., \& Kazdin, A.E. (2009). Identifying evidence-based interventions for children and adolescents using the range of possible changes model: A meta-analytic illustration. Behavior Modification, 33, 583-617.

De Los Reyes, A., \& Prinstein, M. J. (2004). Applying depressiondistortion hypotheses to the assessment of peer victimization in adolescents. Journal of Clinical Child and Adolescent Psychology, 33, 325-335.

De Los Reyes, A., Goodman, K. L., Kliewer, W., \& Reid-Quiñones, K. R. (2008). Whose depression relates to discrepancies? Testing relations between informant characteristics and informant discrepancies from both informants' perspectives. Psychological Assessment, 20, 139-149.

De Los Reyes, A., Henry, D. B., Tolan, P. H., \& Wakschlag, L. S. (2009a). Linking informant discrepancies to observed variations in young children's disruptive behavior. Journal of Abnormal Child Psychology, 37, 637-652.

De Los Reyes, A., Youngstrom, E. A., Pabon, S. C., Youngstrom, J. K., Feeny, N. C., \& Findling, R. L. (invited submission, 2009b). Testing consistencies across and correlates of multiple informant discrepancies in clinical assessments of youths age 11 to 17 years. Manuscript submitted for special section for the Journal of Clinical Child and Adolescent Psychology. 
Ferdinand, R. F., van der Ende, J., \& Verhulst, F. C. (2004). Parentadolescent disagreement regarding psychopathology in adolescents from the general population as a risk factor for adverse outcome. Journal of Abnormal Psychology, 113, 198-206.

Grills, A. E., \& Ollendick, T. H. (2003). Multiple informant agreement and the Anxiety Disorders Interview Schedule for Parents and Children. Journal of the American Academy of Child \& Adolescent Psychiatry, 42, 30-40.

Guion, K., Mrug, S., \& Windle, M. (2009). Predictive value of informant discrepancies in reports of parenting: Relations to early adolescents' adjustment. Journal of Abnormal Child Psychology, $37,17-30$.

Guy, W. (1976). ECDEU Assessment Manual for Psychopharmacology. Washington, DC: DHEW.

Hawley, K. M., \& Weisz, J. R. (2003). Child, parent, and therapist (dis)agreement on target problems in outpatient therapy: The therapist's dilemma and its implications. Journal of Consulting and Clinical Psychology, 71, 62-70.

Holmbeck, G. N. (2002). Post-hoc probing of significant moderational and mediational effects in studies of pediatric populations. Journal of Pediatric Psychology, 27, 87-96.

Israel, P., Thomsen, P. H., Langeveld, J. H., \& Stormark, K. M. (2007). Parent-youth discrepancy in the assessment and treatment of youth in usual clinical care setting: Consequences to parent involvement. European Child \& Adolescent Psychiatry, 16, 138-148.

Johnson, M. K., Hashtroudi, S., \& Lindsay, D. S. (1993). Source monitoring. Psychological Bulletin, 114, 3-28.

Jones, E. E., \& Nisbett, R. E. (1972). The actor and the observer: Divergent perceptions of the causes of behavior. In E. E. Jones, D. E. Kanouse, H. H. Kelly, R. E. Nisbett, S. Valins, \& B. Weiner (Eds.), Attribution: Perceiving the causes of behavior (pp. 7994). Morristown, NJ: General Learning Press.

Kazdin, A. E. (2003). Research design in clinical psychology (4th ed.). Boston: Allyn \& Bacon.

Kendall, P. C. (1994). Treating anxiety disorders in children: Results of a randomized clinical trial. Journal of Consulting and Clinical Psychology, 62, 100-110.

Koenig, K., De Los Reyes, A., Cicchetti, D., Scahill, L., \& Klin, A. (2009). Group intervention to promote social skills in school-age children with pervasive developmental disorders: Reconsidering efficacy. Journal of Autism and Developmental Disorders, 39, $1163-1172$.

Malle, B. F. (2006). The actor-observer asymmetry in attribution: A (surprising) meta-analysis. Psychological Bulletin, 132, 895919.

Mash, E. J., \& Terdal, L. G. (1988). Behavioral assessment of child and family disturbance. In E. J. Mash and L. G. Terdal (Eds.), Behavioral assessment of childhood disorders (2nd ed., pp. 365). New York: Guilford.

Nunnally, J. C., \& Bernstein, I. H. (1994). Psychometric theory (3rd ed.). New York: McGraw-Hill.

Owens, J. S., Goldfine, M. E., Evangelista, N. M., Hoza, B., \& Kaiser, N. M. (2007). A critical review of self-perceptions and the positive illusory bias in children with ADHD. Clinical Child and Family Psychology Review, 10, 335-351.

Panichelli-Mindel, S. M., Flannery-Schroader, E., Kendall, P. C., \& Angelosante, A. G. (2005). Disclosure of distress among anxietydisordered youth: Differences in treatment outcome. Journal of Anxiety Disorders, 19, 403-422.

Pasupathi, M. (2001). The social construction of the personal past and its implications for adult development. Psychological Bulletin, 127, 651-672.

Pelton, J., \& Forehand, R. (2001). Discrepancy between mother and child perceptions of their relationship: I. Consequences for adolescents considered within the context of parental divorce. Journal of Family Violence, 16, 1-15.
Pelton, J., Steele, R. G., Chance, M. W., \& Forehand, R. (2001). Discrepancy between mother and child perceptions of their relationship: II. Consequences for children considered within the context of maternal physical illness. Journal of Family Violence, 16, 17-35.

Rapee, R. M., Barrett, P. M., Dadds, M. R., \& Evans, L. (1994). Reliability of the DSM-III-R childhood anxiety disorders using structured interview: Interrater and parent-child agreement. Journal of the American Academy of Child \& Adolescent Psychiatry, 33, 984-992.

Rogosa, D., Brandt, D., \& Zimowski, M. (1982). A growth curve approach to the measurement of change. Psychological Bulletin, 92, 726-748.

Rogosa, D. R., \& Willett, J. B. (1983). Demonstrating the reliability of the difference score in the measurement of change. Journal of Educational Measurement, 20, 335-343.

Safford, S. M., Kendall, P. C., Flannery-Schroeder, E., Webb, A., \& Sommer, H. (2005). A longitudinal look at parent-child diagnostic agreement in youth treated for anxiety disorders. Journal of Clinical Child and Adolescent Psychology, 34, 747-757.

Shaffer, D., Gould, M. S., Brasic, J., Ambrosini, P., Fisher, P., Bird, H., et al. (1983). A children's global assessment scale (CGAS). Archives of General Psychiatry, 40, 1228-1231.

Silverman, W. K., \& Albano, A. M. (1996). The Anxiety Disorders Interview Schedule for Children (ADIS-C/P). San Antonio, TX: Psychological Corporation.

Silverman, W. K., Kurtines, W. M., Ginsburg, G. S., Weems, C. F., Lumpkin, P. W., \& Carmichael, D. H. (1999a). Treating anxiety disorders in children with group cognitive-behavioral therapy: A randomized clinical trial. Journal of Consulting and Clinical Psychology, 67, 995-1003.

Silverman, W. K., Kurtines, W. M., Ginsburg, G. S., Weems, C. F., Rabian, B., \& Serafini, L. T. (1999b). Contingency management, self-control, and education support in the treatment of childhood phobic disorders: A randomized clinical trial. Journal of Consulting and Clinical Psychology, 67, 675-687.

Silverman, W. K., \& Ollendick, T. H. (2005). Evidence-based assessment of anxiety and its disorders in children and adolescents. Journal of Clinical Child and Adolescent Psychology, 34, 380-411.

Treutler, C. M., \& Epkins, C. C. (2003). Are discrepancies among child, mother, and father reports on children's behavior related to parents' psychological symptoms and aspects of parent-child relationships? Journal of Abnormal Child Psychology, 31, $13-27$.

Wagner, K. D., Berard, R., Stein, M. B., Wetherhold, E., Carpenter, D. J., Perera, P., et al. (2004). A multicenter, randomized, doubleblind, placebo-controlled trial of paroxetine in children and adolescents with social anxiety disorder. Archives of General Psychiatry, 61, 1153-1162.

Weems, C. F., Costa, N. M., Watts, S. E., Taylor, L. K., \& Cannon, M. F. (2007). Cognitive errors, anxiety sensitivity, and anxiety control beliefs: Their unique and specific associations with childhood anxiety symptoms. Behavior Modification, 31, 174-201.

Weems, C. F., Taylor, L. K., Marks, A. B., \& Varela, R. E. (in press). Anxiety sensitivity in childhood and adolescence: Parent reports and factors that influence associations with child reports. Cognitive Therapy and Research.

Weisz, J. R., Jensen Doss, A., \& Hawley, K. M. (2005). Youth psychotherapy outcome research: A review and critique of the evidence base. Annual Review of Psychology, 56, 337-363.

Weisz, J. R., McCarty, C. A., \& Valeri, S. M. (2006). Effects of psychotherapy for depression in children and adolescents: A meta-analysis. Psychological Bulletin, 132, 132-149.

Weisz, J. R., Weiss, B., Alicke, M. D., \& Klotz, M. L. (1987). Effectiveness of psychotherapy with children and adolescents: A meta-analysis for clinicians. Journal of Consulting and Clinical Psychology, 55, 542-549. 